\title{
Incentives and Targeting Policies for Automated Demand Response Contracts*
}

\author{
Marilena Minou $^{\dagger} \quad$ George D. Stamoulis ${ }^{\dagger} \quad$ George Thanos $^{\dagger} \quad$ Vikas Chandan $^{\ddagger}$ \\ ${ }^{\dagger}$ Department of Informatics, Athens University of Economics and Business, Greece \\ ${ }^{\ddagger}$ IBM Research India
}

\begin{abstract}
Demand Response (DR) programs constitute an efficient way to alleviate the problem of peak demand in smart grids. While the potential impact of DR can be significant, its success essentially depends on the participation and responsiveness of consumers. In this paper, we focus on the design of effective contract-based automated DR programs by energy providers that own supportive generators to meet excess demand and employ DR as a means to avoid their costly activation. We derive a theoretically justified formula for the amount of incentives that should be offered to a consumer to accept such a contract. Based on this, we introduce an algorithm for selecting the optimal set of consumers in terms of total incentives. This algorithm is employed under two different policies for restricting (in a different way) the discomfort caused to consumers. We evaluate these policies using real-world data and present interesting insights about the efficient selection of consumers to be targeted for DR and the total amount of incentives offered to them by the provider.
\end{abstract}

\section{INTRODUCTION AND MOTIVATION}

Demand Response (DR) constitutes an effective solution to address the supply-demand imbalances in smart grids [1] and avoid the associated high production costs. However, designing a successful DR program greatly depends on consumers' participation, which in turn is highly affected by the level of inconvenience caused to them during a DR event due to the modification imposed to their consumption patterns [1]. Energy providers offer various types of DR programs with associated incentives to compensate for this inconvenience. Estimating the appropriate amount of incentives needed to motivate consumers to participate in DR is considered a major challenge for DR designers.

In this paper we focus on incentive-based Automated DR (ADR) programs for residential consumers that exploit the use of Direct Load Control enabled appliances. Such programs can be applied by an energy provider to serve the demand up to an upper threshold $Q^{s}$ of daily electricity capacity, above which the marginal cost of supplying is much higher. Although it is common for providers in various countries and contexts of operation to cope with such a situation, our work is motivated by a realisation of this setting that we encountered in Lulea, Sweden, in the context of projects WATTALYST and OPTi. In Lulea, the local provider uses the steelworks excess gas to operate the main production plant as the best choice from both an economic and environmental perspective. However, when this is not sufficient (e.g. at 7-8am in weekdays), the

\footnotetext{
* Work partly supported by European Union's Seventh Framework Programme (FP7/2007-2013) project 288322, WATTALYST and by Horizon 2020 Programme project 649796 , OPTi.
}

provider activates the peak load generators and uses costly supplementary energy production (e.g. via fossil fuels). This results in considerable additional generation cost as well as in a negative environmental impact [2]. By implementing DR programs, such a provider can limit the total daily demand to the base capacity threshold $Q^{s}$ to avoid the operation of its peak production plants.

One of the most important challenges in DR is the uncertainty on the actual load curtailment to be attained by consumer. In ADR there exists an a priori contract-based agreement between the provider and the consumer about the load to be curtailed or interrupted directly by the provider. However, the consumer should be adequately incentivized to adopt this contract. Furthermore, to make such a contract more attractive, it should include terms restricting the discomfort caused to consumers, conferring fairness on them and avoiding negative phenomena such as participation fatigue. In each DR event, the load of the targeted consumers will be curtailed subject to the agreed restrictions of discomfort, but they will be offered incentives according to their contract.

To address these challenges, we build on previous work [3], which deals with incentives for single DR events, and present in this paper a methodology for designing efficient contract-based ADR programs taking into account consumers' preferences and external context, e.g., the weather. We design ADR programs according to which consumers are both compensated for a specified amount of energy curtailment and assigned an optimal schedule, using a consumer net benefit maximization approach. In particular, the key contributions of this paper are:

1) A novel, yet simple and theoretically justified approach to determine the value of incentives to be offered to a consumer in an incentive compatible contract, so that he is incited to opt-in ADR, based on the assessment of each consumer's net benefit loss.

2) An algorithm to select the optimal set of consumers to be targeted for DR and two accompanying policies that restrict the discomfort caused to consumers, thus attaining some fairness among them. One of the policies was introduced in [3] and restricts the imposed reduction in consumption. The other policy is innovative and restricts the reduction in consumer's utility due to DR.

We evaluate and compare these policies, using a small realworld dataset, and assess the applicable trade-offs, e.g. the new policy for targeting consumers leads to lower total incentives offered by the provider to attain the goals of the DR program. 


\section{RELATED WORK}

The literature on DR is already very extensive. Below, we briefly discuss certain articles and real-life examples that are more closely related to our work. A recent work [3] proposed an inclusive DR system that helps an electricity provider to design an effective DR event by analysing its consumers' consumption data and external context. It develops a methodology to estimate consumers' consumption preferences that we also employ in our paper, a greedy algorithm to identify the set of consumers to be targeted, and offers incentives for single DR event. On the contrary, [5] and [6] propose economic models for different types of DR programs by simulating consumers' behavior for different incentives and penalties, while incentivebased consumption scheduling mechanisms are presented in [7]. Moreover, according to empirical work on consumers' selection in [4], consumers prefer simple programs and ADR. In fact, it is shown in [8] that together with incentives, the diversity of contract types is important to foster consumer participation. Moreover, [9] deals with two curtailment contract types: automated contracts that prescribe the load curtailment for each consumer and voluntary contracts that allow curtailment to vary with the consumer's opportunity cost. [10] proposes a contract framework for provisioning DR for ancillary services. In real energy markets, several ADR programs gravitate towards the participation of residential consumers [12]; e.g., ADR programs in California offer generous incentives to customers per $\mathrm{kWh}$ of consumption reduction, particularly to those employing a technology enabling the automated response to DR signals [13].

The aforementioned literature clearly recognizes the significance of incentives and rewards for DR adoption. However, no simple yet effective approach on the formulation of the appropriate incentive-based contracts for engaging users in automated DR programs is presented. Therefore, there is an evident gap on designing ADR-specific approaches that also exploit the capabilities of present and future smart grids and appliances. In the present article, inspired by real-life industrydriven requirements, we address this gap. We develop a theoretically sound methodology for the energy provider to plan ADR programs, while offering the minimum amount of incentives and ensuring fairness with respect to inconvenience among the residential consumers participating in DR.

\section{THE MODEL}

We consider a set of $N$ households served by a single energy provider. Households have already signed contracts, according to which, when targeted for DR, they give up control of specific appliances. Thus, we henceforth view each household as a single consumer that collaborates with the utility as agreed. Our basic timeframe in which DR is applied is a single day divided into timeslots (say 24 hours or 46 -hour periods), indexed as $t \in$ $T:=\{1,2, \ldots, T\}$. This day corresponds to a known context; e.g. warm summer weekday.

\section{A. The Consumers}

Each consumer $i$ operates a set of appliances $A_{i}$, such as air conditioning, refrigerator, television etc. For each appliance $\alpha \in A_{i}$ of consumer $i$ we denote by $q_{i, \alpha, t}$ its power consumption during timeslot $t$ and by $\overrightarrow{q_{i, \alpha}}$ the vector $\left(q_{i, \alpha, t}, \forall t \in T\right)$ of power consumptions, all applying for the day considered. Each consumer $i$ is characterized by the following, all of which correspond to the context of the day considered:

- Utility: In each timeslot $t$, consumer $i$ attains a utility $U_{i, \alpha, t}\left(q_{i, \alpha, t}\right)$ from consuming $q_{i, \alpha, t}$ on appliance $\alpha$.

- Charging tariff: At each timeslot $t$, consumer $i$ is charged according to a given price $p_{t}$, which corresponds to the per unit of consumption charge and is previously announced by the provider. For simplicity we assume that $p_{t}$ is common for all consumers, but may depend on the time-of-day.

- Optimal Consumption: Consumer $i$ has an optimal daily consumption vector and an associated optimal consumption $Q_{i}$ for the day considered. Following the approach of [3] (and other articles of course), we assume that these are derived by maximizing the total net benefit of the consumer; see below.

To express the total utility, i.e. the total satisfaction experienced (or the total value acquired) by a consumer when operating a set of specific appliances, as a function of his overall daily consumption pattern $\overrightarrow{i_{i, \alpha}}$, we follow the methodology of [3]. In particular, the utility for each appliance is modeled according to the categorization of appliances by [11] and the corresponding utility functions introduced therein, which are continuously differentiable concave functions of $q_{i, \alpha, t}$. Concavity is in line with diminishing additional satisfaction from an additional unit of energy, as the total consumption increases. Furthermore, the total utility attained is the weighed sum of the per appliance utilities; note that $U_{i, \alpha, t}\left(q_{i, \alpha, t}\right)$ as defined above incorporates the weight assigned by consumer $i$ to appliance $\alpha$. Summing these $U_{i, \alpha, t}\left(q_{i, \alpha, t}\right)$ for the various appliances and timeslots, we obtain the total daily utility. Given that the energy tariff scheme is known and declared above, each consumer $i$ is assumed to act rationally by choosing that consumption schedule that maximizes his total Net Benefit $\left(N B_{i}\right)$, i.e. the utility gained minus the monetary charge.

\section{Consumer $i$ 's objective (max net benefit):}

$$
\arg \max _{q_{i, \alpha}} \sum_{t \in T} \sum_{\alpha \in A_{i}} U_{i, \alpha, t}\left(q_{i, \alpha, t}\right)-\sum_{t \in T} p_{t} Q_{i, t}
$$

where $Q_{i, t}=\sum_{\alpha \in A_{i}} q_{i, \alpha, t}$. We denote also as $U_{i, t}\left(Q_{i, t}\right)=$ $\sum_{\alpha \in A} U_{i, \alpha, t}\left(q_{i, \alpha, t}\right)$ the utility obtained by consumer $i$ from the consumption over all appliances in timeslot $t$ and as $U_{i}\left(Q_{i}\right)=$ $\sum_{t \in T} U_{i, t}\left(Q_{i, t}\right)$ the total utility obtained from the consumption over all timeslots. Note that both utility and net benefit are expressed in monetary units.

\section{B. The Energy Provider}

We consider the case of an energy provider who owns supportive peak load generators, activated only when the base plant production cannot meet the excess demand of its customers. In particular, we assume that there is an upper threshold $Q^{s}$ of electricity production in each day, above which the marginal cost of production is very increased due to the activation of supplementary energy based generators. Thus, the operator wishes to restrict production below $Q^{s}$. Regarding production cost, we assume that the provider is characterized by a cost 
function $C(Q)$, where $Q=\sum_{i \in N} Q_{i}$ and $Q_{i}=\sum_{t \in T} Q_{i, t}$. The function specifies the cost for the provider to provide a daily $Q$ amount of power. We assume that the cost function $C(Q)$ is convex and increasing in $Q$ (in accordance to [11]) as long as $Q \leq Q^{s}$. Note that in general the cost of operating the basic power plant should be estimated per timeslot $t$ rather than on a daily basis. Also, the production upper threshold should be taken to apply for each timeslot. In our setting, for simplicity we only consider the daily constraint of $Q^{s}$, which is less restrictive, as well as a daily total production cost. These assumptions apply e.g. when the provider possesses some storage capabilities.

As the provider aims to limit the production below the threshold $Q^{s}$, he may have to resort to DR, to limit consumers' total daily demand accordingly. Since this threshold is strict, automated DR, which imposes surely the necessary reductions in the demand to the targeted consumers, is the most appropriate approach. Whenever a DR event is necessary (see Section IV), the provider has to solve the following optimization problem:

Energy Provider's objective:

$$
\arg \max _{I_{i}, Q_{i, t}} \sum_{i \in N} \sum_{t \in T} p_{t} \hat{Q_{i, t}}-C\left(\sum_{i \in N} \hat{Q_{i}}\right)-\sum_{i \in N} I_{i}
$$

such that

$$
\sum_{i \in N} \hat{Q}_{i} \leq Q^{s}
$$

where $\hat{Q}_{i}=\sum_{t \in T} \hat{Q_{i, t}}$. Recall that $Q_{i, t}$ denotes the unconstrained total consumption of consumer $i$ if no reduction is imposed, while we use "hat" to denote the new consumption schedules after the reductions in demand are enforced by the provider to selected consumers targeted for DR. Also, $I_{i}$ denotes the incentives offered to consumer $i$ if targeted for this DR event. In cases where the constraint on the total demand (3) holds with equality (which we will assume when applying the targeting algorithm presented below) both the total revenue and the total cost of the provider are fixed. Therefore, the optimal boundary solution of (2) is that of the problem of minimizing the total incentives, i.e.:

$$
\arg \min _{I_{i}} \sum_{i \in N} I_{i}
$$

Note that, as explained below, the incentives are already prescribed in the contracts, and in fact they should be defined in such a way that the consumers accepting the contracts are adequately compensated whenever targeted for DR. Therefore, the above problem amounts to targeting for DR the consumers needing the least total incentives to meet the threshold on the total demand. In fact, the provider should verify that the cost savings attained due to DR exceed the losses due to the incentives and to selling less energy.

\section{InCENTIVES AND TARgeting Policies}

In this section, we specify $i$ ) the DR incentives that should be included as terms in the contracts of the consumers engaged in DR and ii) which consumers should be targeted in a day where DR is necessary for the provider. To achieve these we make the following assumptions; within the time horizon of a day, for which DR planning is to be performed, the consumers can shift load between different slots. However, they cannot shift load between different days, since by assumption consumers are characterized by an optimal consumption per day, which they choose for themselves as a result of maximixing their net benefit $N B_{i}$ as per (1). Hence, any deviation from this consumption is bound to reduce the net benefit obtained by the consumer. In fact, in the case of DR, a targeted consumer is mandated to consume less than his optimal daily quantity. This results in a lower net benefit as well as in a utility value too, due to the inconvenience caused to him. To this end, in order for a consumer to be convinced to participate in an ADR program, i.e. to sign the relevant contract granting the operator the right to control the appliances in consumer's premises and impose a modified consumption schedule whenever he is targeted for DR, the provider should offer him monetary incentives that correspond to his loss of net benefit. Thus, rational consumers would become indifferent to the changes in their consumption. In other words, the incentives offered to consumers should equal their total net benefit loss due to the reduction in consumption:

$$
I_{i}=N \text { Bloss }_{i}=N B_{i}-\hat{N B_{i}}
$$

where $\hat{N B_{i}}<N B_{i}$. Note that $\hat{N B_{i}}$ expresses the net benefit obtained by consumer $i$ under the new consumption schedule, that is, after the reduction in demand is imposed by the energy provider. Thus, the provider should endeavor DR contracts that are incentive compatible, i.e. they serve the interests of both the consumer and the provider, both of whom are better off and thus have the incentive to adopt the contract. Moreover, as already explained a targeted user also incurs a loss in utility. Hence, the ADR contract should also guarantee limits to the possible inconvenience. Therefore, we conclude that in order for ADR to be attractive to users, the contract terms should specify beforehand i) the incentives' calculation methodology, and particularly that whenever targeted for DR the consumer will be compensated the amount equal to the loss of net benefit caused, and $\mathrm{ii}$ ) the maximum possible reduction in the inconvenience, about which we consider two different policies below. In particular, the provider can either enforce a maximum percentage reduction in consumption $\eta_{\max }$ that can be imposed to each of the targeted consumers, or alternatively define the maximum reduction in utility $\eta_{\max _{u}}$ suffered per targeted consumer.

The provider now is faced with the following situation. He is assumed to have determined in advance the maximum amount of energy $Q^{s}$ that he can supply; he charges consumers with price $p_{t}$ per unit of consumption and maintains knowledge (either full or limited) of the total power demand $Q_{i}$, of the vector of optimal consumption $\overrightarrow{Q_{i}}:=\left\{Q_{i, t}, \forall t \in T\right\}$ and of the utility function of consumer $i, U_{i, t}\left(\vec{Q}_{i, t}\right):=\left\{U_{i, t}\left(Q_{i, t}\right), \forall t \in T\right\}$.

The consumers, which are likely to be targeted, are those that have already signed contracts with the provider, which include their enrollment in an ADR program and grant permission for their election in order to be targeted for DR. The terms of these contracts are defined as above, so that they are incentive compatible. For simplicity, we henceforth assume that all consumers have signed such contracts, and are eligible to be targeted.

When it is predicted that the total demand will exceed the threshold $Q^{s}$, the energy provider activates the ADR programs 
and resorts to targeting a subset of the consumers in order to offer the minimum total incentives to restrict total demand at or below $Q^{s}$, while also abiding with the terms of the signed contracts. To this end, the provider should employ the corresponding selection algorithm associated with each of two policies described in the sequel.

\section{A. Policy 1: Constrained reduction in consumption}

Here, the provider defines a priori the maximum percentage reduction in consumption $\eta_{\max _{q}}$, as a fraction of consumers' optimal consumption $Q_{i}, \forall i \in N$. To simplify our discussion, we assume that $\eta_{\max _{q}}$ is the exact percentage reduction to be imposed to each targeted consumer. Then, by applying Algorithm 1 the set of consumers to be targeted is extracted. The main idea is that the consumers are targeted in increasing order of the incentives (i.e., reduction in net benefit) per unit of consumption reduction applicable for this value of $\eta_{\max _{q}}$.

Algorithm 1: Consumer Selection

STEP 1: Define the required reduction to be achieved as:

$$
\Delta Q=\sum_{i \in N} Q_{i}-Q^{s}
$$

Continue only if this is positive.

STEP 2: For each consumer $i$ define the reduction in consumption as a percentage of the optimal consumption $\Delta Q_{i}=$ $\eta_{\max _{q}} Q_{i}$; also define the new consumption as $\hat{Q}_{i}=Q_{i}-\Delta Q_{i}$ to be consumed.

STEP 3: For each consumer $i$ find $\hat{Q}_{i}$ and the vector $\left\{\hat{Q_{i, t}}, \forall t \in\right.$ $T\}$ that maximizes his $\hat{N B_{i}}$, i.e. for each consumer $i$ solve:

$$
\arg \max _{\overrightarrow{q_{i, \alpha}}} \sum_{t \in T} \sum_{\alpha \in A_{i}} U_{i, \alpha, t}\left(q_{i, \alpha, t}\right)-\sum_{t \in T} p_{t} \hat{Q_{i, t}}
$$

such that $\sum_{t \in T} \sum_{\alpha \in A_{i}} q_{i, \alpha, t}=\hat{Q}_{i}$

STEP 4: For each consumer $i$ : a) calculate the net benefit loss as per (5) $b$ ) set the incentives $I_{i}$ equal to the net benefit loss NBLoss L $_{i}$

STEP 5: For each consumer $i$ estimate the incentive that corresponds to one unit reduction in consumption as $u I_{i}=\frac{I_{i}}{\Delta Q_{i}}$. STEP 6: Sort consumers in ascending order of the value of $u I_{i}$. We refer to this set by $S$. Set $j=1$.

STEP 7: Set the temporary decision set $T S$ of the targeted consumers as $\{j, j+1, \ldots, j+N-1\}$. Set $j=j+1$.

STEP 8: Repeat step 6 until $\sum_{i \in T S}\left(\hat{Q}_{i}\right) \geq \Delta Q$

STEP 9: Make the necessary adjustments for the last selected consumer.

STEP 10: The final set of targeted consumers is given by $T S$ and the respective consumption patterns are those derived in Steps 3 and 9 . The rest of the consumers are not targeted thus maintaining their unconstrained optimal consumption pattern.

Note, that the last selected consumer is not always assigned a reduction equal to $\hat{Q_{i, t}}$, but the remaining amount of the required reduction so that we attain the exact consumption threshold. For this consumer, in Step 9 of the algorithm, Steps 3 and 4 should be ran again to calculate his net benefit loss to be incurred and the necessary incentives.
Although the provider decides on the value of $\eta_{\max }$ in such a way that the algorithm results in a subset of consumers targeted, there is a lower threshold $\eta_{\text {min }_{q}}$, under which the proposed reductions in consumers' consumption do not achieve the provider's goal with regard to (6) meaning that $\eta_{\max _{q}}$ must satisfy some feasibility condition. For the required reduction in demand $\Delta Q=\sum_{i \in N} \Delta Q_{i}$ we have that

$$
\Delta Q \leq \eta_{\max _{q}} \sum_{i \in N} Q_{i}
$$

Using (8) we establish that the percentage of reduction in consumption should be (at least) equal to:

$$
\eta_{\text {min }_{q}}=\frac{\sum_{i \in N} Q_{i}-Q^{s}}{\sum_{i \in N} Q_{i}}=1-\frac{Q^{s}}{\sum_{i \in N} Q_{i}}
$$

Hence the condition (9) leads to a feasible solution of the problems (2) and (7).

\section{B. Policy 2: Constrained reduction in utility}

So far, we have assumed that for each consumer $i$ the permissible reduction in consumption is expressed in the contract as a percentage $\eta_{\max _{q}}$ of the optimal consumption. This was also the assumption made in [3] in order for the provider to indirectly enforce some limit to the inconvenience caused. However, it is plausible that even a small reduction of the energy consumed can cause great inconvenience, especially in cases where consumers appear to be less flexible to adjustments in their schedule. Thus, in such cases, constraining the percentage reduction in the utility gained by each consumers is expected to be preferable in terms of both the inconvenience caused and the total incentives to be offered, since the smaller the difference in utility, the lower the inconvenience cost that the consumers have to incur. To this end, for this algorithm the selection criterion differs only in STEPS 2 and 3 as follows:

STEP 2: For each consumer $i$ define the reduction in utility as a percentage of the utility obtained under the optimal consumption schedule $\Delta U_{i}=\eta_{\max _{u}} U_{i}\left(Q_{i}\right)$.

STEP 3: For each consumer $i$ find $\hat{Q}_{i}$ and the vector $\left\{\hat{Q_{i, t}}, \forall t \in T\right\}$ that maximises his $\hat{N B_{i}}$, i.e. for each consumer $i$ solve:

$$
\arg \max _{q_{i, \alpha}} \sum_{t \in T} \sum_{\alpha \in A_{i}} U_{i, \alpha, t}\left(q_{i, \hat{\alpha}, t}\right)-\sum_{t \in T} p_{t} \hat{Q_{i, t}}
$$

such that $\sum_{t \in T} \sum_{\alpha \in A_{i}} U_{i, \alpha, t}\left(q_{i, \alpha, t}\right)=U_{i}\left(Q_{i}\right)-\Delta U_{i}$

A similar approach involves the enforcement of the reduction in terms of net benefit rather than utility, which would ensure also fairness on the selection of consumers without though guaranteeing the percentage reduction in consumers' discomfort. Further investigation of this approach is left for future research.

\section{EXPERIMENTAL EVALUATION}

To experimentally evaluate our approaches we use real consumption data from 6 households in India. The data constitutes of sensor readings at a granularity of ten seconds. We distinguish two cases with regard to the type of the available data. First, we 
assume that appliance level measurements for four different appliances are available (air-conditioner, fridge, washing machine and television). Second, the provider's information is limited to consumption data concerning only the most important devices, i.e. those that consume large loads; hence we consider only measurements for the air-conditioner and fridge that comprise the largest portion of the total load. The readings in both cases are extracted for a given context, that is weekday 11 April 2014, and are used as input to the methodology described in Section 3.2 of [3] to extract knowledge on each consumer's utility functions and the corresponding weights, indicating the importance that the consumer places on each specific appliance and the flexibility in altering its operation. For each day and consumer, the recorded data is used to obtain consumption in Wh for each time slot with a duration of 6 hours during the day. So, we obtain the optimal consumption $Q_{i}$ of consumer $i$.

\section{A. Evaluation of Policy 1: Full vs Limited information}

Say that the energy provider wishes to narrow the total demand by a value $\Delta Q$ that amounts to $10 \%$ of the unconstrained total optimal consumption and define the value of $\eta_{\max _{q}}$. Following (9), the value of $\eta_{\max _{q}}$ must be greater or equal to $10 \%$. Hence, we have taken three different values for $\eta_{\max _{q}}$ to investigate the results regarding the incentives offered, the social welfare achieved and the set of selected consumers. For conciseness, Table I depicts the results of the selection algorithm for two values of $\eta_{\max _{q}}$. Consumers are listed in ascending order of $u I_{i}$ (in Indian Rupee $(₹)$ ) together with their consumption reduction $\Delta Q_{i}$ (in Wh) and utility reduction $\Delta U_{i}$ (also expressed in ₹).

The results indicate that for different values of $\eta_{\max }$, the number of selected consumer decreases and therefore the incentives offered by the provider. In particular, in the case of full information (Table Ia), we observe that a small increase of $\eta_{\max _{q}}$ leads to the same number and set of selected consumers but with lower amount of incentives, which is anticipated as the constraints are relaxed and the algorithm can impose greater reduction to those consumers with lower value of $u I_{i}$ (which however depends on $\eta_{\max }$ ) and avoid allocating large reductions to the "expensive" consumers. If we increase the value of $\eta_{\max _{q}}$ to $20 \%$, then the algorithm results in selecting only 3 out of 6 consumers to target for DR, yielding in lower total incentives equal to $162.12 ₹$.

Similar outcomes arise in the case of limited information in Table Ib. Here, the increase of the value of $\eta_{\max _{q}}$ has a direct impact on the number of selected consumers and the total incentives. A further increase of $\eta_{\max _{q}}$ to $20 \%$, leads to even better results in terms of both the number of selected consumers (only 2) and the total incentives to be offered (173.71₹). However, in this case more incentives are required than when full information is available. This is due to the inelastic nature of the particular type of devices, their operational constraints and the context under which they operate. Still, it is entirely up to the provider to decide whether investing in appliance level meters is more economically efficient in the long run than the cost of providing incentives to his consumers.

\section{B. Evaluation of Policy 2: Full vs Limited Information}

Here, we consider that the energy provider wishes to narrow the total demand by $\Delta Q=25 \%$, without reducing consumers' utility more than $\eta_{\max _{u}}=5 \%$. Apparently, for such small value of $\eta_{\max _{u}}$ the problem is very restricted and the optimal solution would be to select all consumers in order to achieve provider's objective. Nevertheless, both under full and limited information, the algorithm selects 4 out 6 consumers to target for DR (see Table II). Again, for limited knowledge, the algorithm leads to a higher total amount of incentives offered, which is of course reasonable, due to the type of loads that are considered and the restrictions in the consumption schedules to be proposed.

\section{Comparison of the policies}

So far we can deduce that Policy 2 selects the optimal set of consumers to be targeted with the minimum possible incentives without the need to apply large reductions in consumers' utility values. However, a comparison of the two policies with $\eta_{\max _{q}}=\eta_{\max _{u}}$ would not be fair, as despite the fact that both policies aim at solving the same problem, these percentages are included in different constraints. In this context, another important performance index to be reckoned is the number of consumers to be targeted. To this end, we have defined a specific setup in order to evaluate their performance, by considering a case where the provider has full knowledge and both policies would target the same number of consumers. Due to the small total number of consumers' data available, we introduce in the sequel values (e.g. in reduction of consumption and utility) that do not correspond to real scenarios but serve our scope to provide some insights concerning the best choice of policies employed. Thus, say that the energy provider aims at reducing the total demand by $20 \%$. To achieve that, consumers' consumption is reduced by $\eta_{\max _{q}}=30 \%$ under Policy 1 and their utility by $\eta_{\max _{q}}=10 \%$ under Policy 2 .

The outcomes suggest that if both policies select the same number of consumers to target (thus providing a fair basis for comparison), even for more restrictive values of $\eta_{\max _{u}}$ Policy 2 outperforms Policy 1 in terms of incentives (123.75₹, 86.55₹for Policy 1 and 2 resp.). This is due to the fact that even a small reduction of the energy consumed can cause great inconvenience, which in turn requires high incentives to compensate the consumer. By employing Policy 2, the energy provider acquires a more clear insight of the consumers behaviour and their corresponding discomfort levels and therefore selects the set of consumers targeted more effectively. Another observation refers to the sorting list of consumers. One would expect that the process of ordering would provide the same results, since both algorithms follow the same sorting criterion, but this is not always the case as the decrease imposed to the consumers in their consumptions is estimated differently in each case, hence the values of $u I_{i}$ and $I_{i}$ may vary as well. Although in the example the same number of consumers is targeted, the sets of targeted consumers differs. Thus, in an environment of similar characteristics, the provider applying ADR and aiming to minimize the total incentives should choose Policy 2, even if he has to commit to a strict constraint on utility loss (i.e. to a low value of $\eta_{\text {max }_{u}}$ ) to make the contracts more attractive. 
TABLE I: Policy 1: Full vs Limited Information for $\Delta Q_{d}=10 \%$

(A) Policy 1: Full Information

(B) Policy 1: Limited Information

\begin{tabular}{|c|c|c|c|c|c|c|c|c|c|c|c|c|c|}
\hline & \multicolumn{3}{|c|}{$\eta_{\max , q}=12 \%$} & \multicolumn{3}{|c|}{$\eta_{\max , \mathrm{q}}=15 \%$} & & \multicolumn{3}{|c|}{$\eta_{\max , q}=12 \%$} & \multicolumn{3}{|c|}{$\eta_{\max , \mathbf{q}}=15 \%$} \\
\hline Consumer & $\mathbf{I}_{\mathbf{i}}(\bar{₹})$ & $\Delta Q_{\mathrm{i}}(\mathrm{Wh})$ & $\Delta U_{i}$ (₹) & $\overline{I_{i}(₹)}$ & $\Delta Q_{\mathrm{i}}(\mathrm{Wh})$ & $\Delta \bar{U}_{i}($ ₹ $)$ & Consumer & $\overline{I_{i}(₹)}$ & $\Delta Q_{\mathrm{i}}(\mathrm{Wh})$ & $\Delta \mathrm{U}_{\mathrm{i}}(\mathrm{₹})$ & $\overline{I_{i}(₹)}$ & $\Delta Q_{\mathrm{i}}(\mathrm{Wh})$ & $\Delta \mathrm{U}_{\mathrm{i}}(\mathrm{\gtrless})$ \\
\hline 4 & 23.57 & 4476.13 & 26.13 & 23.93 & 4323.54 & 26.14 & 5 & 23.30 & 3509.99 & 25.31 & 23.80 & 3390.33 & 25.31 \\
\hline 5 & 22.12 & 3509.99 & 25.16 & 22.62 & 3390.33 & 25.19 & 1 & 151.90 & 20287.97 & 163.52 & 152.99 & 19596.33 & 163.52 \\
\hline 1 & 134.98 & 20287.97 & 146.71 & 135.07 & 19596.33 & 146.79 & 6 & 99.58 & 12523.48 & 106.75 & 82.82 & 13028.23 & 106.75 \\
\hline 6 & 69.52 & 12827.32 & 103.16 & 65.39 & 13791.21 & 103.17 & 3 & 29.59 & 3575.18 & 31.19 & 0 & 0 & 0 \\
\hline 3 & 0 & 0 & 0 & 0 & 0 & 0 & 4 & 0 & 0 & 0 & 0 & 0 & 0 \\
\hline 2 & 0 & 0 & 0 & 0 & 0 & 0 & 2 & 0 & 0 & 0 & 0 & 0 & 0 \\
\hline Total Incentives (₹) & \multicolumn{3}{|c|}{250.18} & \multicolumn{3}{|c|}{247.02} & Total Incentives $(₹)$ & \multicolumn{3}{|c|}{304.37} & \multicolumn{3}{|c|}{259.61} \\
\hline
\end{tabular}

TABLE II: Policy 2: Full vs Limited Information for $\Delta Q_{d}=25 \%$ and $\eta_{\max _{\mathbf{u}}}=\mathbf{5} \%$

(A) Policy 2: Full Information

\begin{tabular}{|c|c|c|c|}
\hline Consumer & $\mathbf{I}_{\mathbf{i}}(\bar{₹})$ & $\Delta \mathbf{Q}_{\mathbf{i}}(\mathrm{Wh})$ & $\Delta \mathbf{U}_{\mathbf{i}}(\bar{₹})$ \\
\hline 5 & 41.83 & 5124.57 & 25.30 \\
\hline 4 & 23.89 & 2813.56 & 26.13 \\
\hline 3 & 43.34 & 4058.66 & 31.10 \\
\hline 2 & 7.73 & 1151.87 & 24.89 \\
\hline 6 & 0.00 & 0.00 & 0.00 \\
\hline 1 & 0.00 & 0.00 & 0.00 \\
\hline Total Incentives (₹) & \multicolumn{4}{|c}{116.80} \\
\hline
\end{tabular}

\section{CONCLUSIONS AND FUTURE WORK}

In this paper we developed a methodology to assist the design of effective contract-based ADR programs by energy providers in order for them to avoid the costly activation of extra (peak load) generators. We derived a theoretically justified methodology for the calculation of incentives to be offered to a consumer in order to accept such a contract and introduced an algorithm for selecting the optimal set of consumers. This algorithm is employed under two different policies for restricting (in a different way) the discomfort caused to consumers. The results have shown that for increasing values of the permissible reduction in comfort per consumer, the number of selected consumers decreases and so do the total incentives required to be granted by the provider to meet his goal. In addition, the policy that restricts directly the reduction in each consumer's utility selects the optimal set of consumers with a lower amount of incentives without imposing large reductions in utility values.

Moreover, in the case of limited information higher incentives are required than when full information is available. This is due to the inelastic nature of the particular type of devices, their operational constraints and the context under which they operate. On the contrary, in the full information case, small domestic appliances, e.g. washing machines, are more flexible in changes in their consumption pattern thus resulting in lower required incentives. However, whether the benefits of the knowledge of full information justify the cost of deploying extra sensors is not apparent. A detailed cost/benefit analysis is needed for the provider to decide whether investing in appliance level meters is more economically efficient in the long run than the cost of providing higher incentives to his consumers due to incomplete information. With the decreasing cost of sensors and the continuous growth of Advanced Metering Infrastructure (AMI) deployment, it is expected that investing in a few additional sensors, yet only for appliances with significant consumption, can be beneficial.

In our future work, we intend to validate our model on the
(B) Policy 2: Limited Information

\begin{tabular}{|c|c|c|c|}
\hline Consumer & $\mathbf{I}_{\mathbf{i}}(\bar{₹})$ & $\mathbf{\Delta} \mathbf{Q}_{\mathbf{i}}(\mathrm{Wh})$ & $\mathbf{\Delta} \mathbf{U}_{\mathbf{i}}(\bar{₹})$ \\
\hline 4 & 67.68 & 6980.62 & 42.21 \\
\hline 5 & 41.01 & 3958.40 & 25.32 \\
\hline 3 & 30.24 & 1714.88 & 31.20 \\
\hline 2 & 20.12 & 494.76 & 24.93 \\
\hline 1 & 0.00 & 0.00 & 0.00 \\
\hline 6 & 0.00 & 0.00 & 0.00 \\
\hline Total Incentives (₹) & \multicolumn{3}{|c|}{159.05} \\
\hline
\end{tabular}

basis of a larger set of available data from real consumers, so that we our evaluation can match more closely real life conditions. Furthermore, we plan to include certain terms in the contract to deal with the possibility of fatigue of targeted consumers, by restricting the total number of times they are targeted e.g. within a month, or by increasing by a small factor the incentives of frequently targeted consumers.

\section{REFERENCES}

[1] U. D. of Energy, "Benefits of demand response in electricity markets and recommendations for achieving them," 2006.

[2] M. Minou, G. Thanos, M. Vasirani, T. Ganu, M. Jain, and A. Gylling, "Evaluating demand response programs: Getting the key performance indicators right," International Workshop on Demand Response, 2014.

[3] V. Chandan, T. Ganu, T. K. Wijaya, M. Minou, G. Stamoulis, G. Thanos, and D. P. Seetharam, "iDR: consumer and grid friendly demand response system," in Proceedings of the 5th international conference on Future energy systems, (pp. 183-194), ACM.

[4] E. Dütschke and A.-G. Paetz, "Dynamic electricity pricing?which programs do consumers prefer?"” Energy Policy, vol. 59, 2013.

[5] H. Aalami, M. P. Moghaddam, and G. Yousefi, "Demand response modeling considering interruptible/curtailable loads and capacity market programs," Applied Energy, vol. 87, no. 1, pp. 243-250, 2010.

[6] M. P. Moghaddam, A. Abdollahi, and M. Rashidinejad, "Flexible demand response programs modeling in competitive electricity markets," Applied Energy, vol. 88, no. 9, pp. 3257-3269, 2011.

[7] A.-H. Mohsenian-Rad, V. W. Wong, J. Jatskevich, and R. Schober, "Optimal and autonomous incentive-based energy consumption scheduling algorithm for smart grid," in Innovative Smart Grid Technologies (ISGT), 2010, pp. 1-6, IEEE, 2010.

[8] X. He, N. Keyaerts, I. Azevedo, L. Meeus, L. Hancher, and J.-M. Glachant, "How to engage consumers in demand response: a contract perspective," Utilities Policy, vol. 27, pp. 108-122, 2013.

[9] K. M. Daniels and R. Lobel, "Demand response in electricity markets: Voluntary and automated curtailment contracts," 2014.

[10] T. Haring, G. Andersson, "Contract design for demand response," 2014.

[11] N. Li, L. Chen, and S. H. Low, "Optimal demand response based on utility maximization in power networks," in Power and Energy Society General Meeting, 2011 IEEE, pp. 1-8, IEEE, 2011.

[12] B. Feldman, B. Lockhart, "Automated Demand Response," Navigant Research, 2014.

[13] Energy.Gov: Office of Energy Efficiency and Renewable Energy, "Energy Incentive Programs, California". 